\title{
Transient Primary Bone Marrow Edema Syndrome - A Synthesis
}

\author{
Nissim Ohana ${ }^{1}$, Dimitri Sheinis ${ }^{2}$, Daniel Benharroch ${ }^{3, *}$ \\ ${ }^{1}$ Department of Orthopeadic Surgery, Meir Medical Center, Kfar Saba and Faculty of Medicine, Tel Aviv University, Tel Aviv, Israel \\ ${ }^{2}$ Department of Orthopeadic Surgery, Soroka University Medical Center, and Faculty of Health Sciences, Ben Gurion University of the \\ Negev, Beer-Sheva, Israel \\ ${ }^{3}$ Independent Physician, Department of Pathology, Soroka University Medical Center and Faculty of Health Sciences, Ben Gurion University \\ of the Negev, Beer-Sheva, Israel
}

Email address:

danielbenharroch1@gmail.com (D. Benharroch)

${ }^{*}$ Corresponding author

\section{To cite this article:}

Nissim Ohana, Dimitri Sheinis, Daniel Benharroch. Transient Primary Bone Marrow Edema Syndrome - A Synthesis. American Journal of Internal Medicine. Vol. 7, No. 2, 2019, pp. 27-32. doi: 10.11648/j.ajim.20190702.11

Received: March 4, 2019; Accepted: April 4, 2019; Published: May 15, 2019

\begin{abstract}
A complex and often confusing nomenclature is currently used for relatively rare syndromes characterized by sudden onset of joint pain in the lower limbs, absence of trauma, bone marrow edema and its resolution, both confirmed by the MRI, as well as a self-limiting course. These include transient osteoporosis of the hip (TOH), of the knee (TOK), of the foot and ankle and transient bone marrow edema syndrome. Our purpose was to review the literature in order to substantiate the hypothesis that these apparently different conditions may be synthesized into a single disease entity, termed transient primary bone marrow edema syndrome (TPBMES). Of a total of 546 patients scrutinized, 342 had TOH, 105 had TOH of pregnancy, and 49, mainly females, showed transient foot-and-ankle osteoporosis. TPBMES occurred also with systemic osteoporosis or in a migratory pattern. The six proposed subsets of TPBMES have in common a MRI-based diagnosis and remission, as well as a self-limiting course. Thus, the hypothesis of a single disease entity is sustainable. We conclude that the education of the medical profession regarding this rare disease should expand. The causes of the prolonged symptoms seen in the systemic osteoporosis and migratory subsets warrant further studies. The efficiency of our suggested modality of management should be validated in a large cohort.
\end{abstract}

Keywords: Joint Pain, Bone Pain, Transient Bone Marrow Edema Syndrome, Systemic Osteoporosis, Migratory Osteoporosis, Bisphosphonates, Aledronate, Synthesis

\section{Introduction}

Bone marrow edema (BME) represents an increase of fluid content in the marrow. This non-specific MRI finding is typically the result of injury or osteoporosis, but may also be associated with several other conditions [1]. "Transient bone marrow edema syndrome" (TBMES) is a term that was first coined by Wilson et al. in 1988 to describe an alteration of the bone marrow causing joint pain in the hip or knee. Patients' MR imaging showed a combination of a regional subtle decrease of bone marrow signal intensity on Tlweighted images and a corresponding increased signal intensity on T2-weighted images, without evidence of a mass
[2]. TBMES is a self-limiting condition of unknown etiology, which usually affects middle-aged individuals and may last for 3-9 months [3].

Transient osteoporosis of the hip (TOH), of the knee (TOK) and of the foot and ankle, also known collectively as transient regional osteoporosis or idiopathic transient osteoporosis, are all characterized by BME [4-6]. Transient osteoporosis of the hip was first described in 1959 in women in the third trimester of their pregnancy by Curtiss and Kincaid [7], who dubbed it transient demineralization of the hip. In 1968 it was characterized by Lequesne as TOH, 
because of periarticular osteopenia observed 3-6 weeks after the onset of symptoms [8] Subsequent reports have referred to the syndrome by numerous names, including transient osteoporosis, transitory demineralization, migratory osteolysis, regional migratory osteoporosis, idiopathic regional osteoporosis, transient painful osteoporosis, roentgenologic transient osteoporosis, transient osteopenia, algodystrophy, idiopathic transient osteoporosis, and painful regional osteoporosis $[9,10]$. Transient osteoporosis is a selflimiting skeletal condition, the etiology and pathogenesis of which are poorly understood. This relatively rare cause of acute joint pain affects mostly males between the fourth and the sixth decade of life $[1,5,9]$. It presents spontaneously with sudden-onset of pain in one or more joints, gradually resolving within 6-8 months $[4,9,10]$. It is associated with a temporary osteopenia and joint effusion in the region, in the absence of other recognizable causes of synovial fluid or bone abnormalities [4, 5, 10]. Management involves symptomatic treatment and avoidance of weight bearing and persistent efforts to reduce the risk of fracture until the osteoporosis resolves. The alleviation of symptoms is accompanied by remineralization of the affected regions. Differential diagnosis includes tumor, inflammatory arthritis, infection, reflex sympathetic dystrophy, stress fractures, and avascular necrosis (AVN) [4].

In some cases, the condition reappears in a different region of the same joint, or in other ipsilateral or contralateral joints. These conditions are termed "migratory transient osteoporosis" or "regional migratory osteoporosis". These patients also display the same signs of BME on the MRI [1, $11,12]$.

The MRI has become the tool of choice for early diagnosis of $\mathrm{TOH}$, which is based on the detection of BME $[2,3,5,12]$. However, BME may also be associated with other conditions, such as epiphyseal stress fractures and avascular necrosis (AVN) of the femoral head. Avascular necrosis, which is a more common cause of acute hip pain than $\mathrm{TOH}$, is a progressive condition resulting from interrupted blood supply to the femoral head and causing hip joint deformity. Despite the marked differences between the two conditions, TOH and AVN may appear similarly on radiography at early stages [10]. Therefore, the distinction between transient osteoporosis and AVN is of the utmost importance. Whereas $\mathrm{AVN}$ is a progressive, irreversible disease that requires early diagnosis and surgical intervention, non-progressive conditions such as $\mathrm{TOH}$ resolve spontaneously $[1,5,9]$.

Because of the similarity in clinical and imaging findings, we hypothesize that the above mentioned types of transient osteoporosis and transient BME are in fact different subsets of a single disease entity, namely the transient primary bone marrow edema syndrome (TPBMES), which is distinct from AVN. To validate this hypothesis, we reviewed the literature for reports on patients presenting with joint pain in the lower limbs with transient BME, as diagnosed by MRI. The synthesis of the symptoms and signs reviewed suggests that TPBMES is indeed a single disease entity.

\section{Methods}

\subsection{Overview}

A retrospective review of collected articles in English was performed. No experimental investigations on human subjects or animals were performed by any of the co-authors, and, therefore, an IRB approval was not requested. Reviewed papers were included if they describe patients with complaints of lower limb joint pain and with an MRI-based diagnosis of transient bone marrow edema. Only older studies had not used MRI the $[13,14]$. Single or isolated case reports were excluded. Furthermore, patients, hereby scrutinized were required to comply with the inclusion and exclusion criteria, as listed in the study Abstract. Our source was the NCBI, randomly searched for papers published during the last three decades, and using the search terms "transient osteoporosis of the hip", "primary bone marrow edema" and equivalent terms.

\subsection{Tables}

An all-inclusive review table (not shown) was structured for a wide set of patients and was then used to generate tables featuring each of the suggested TPBMES subtypes. Two further tables are devoted to complex TPBMES with either systemic osteoporosis or the migratory variant. The TPBMES subtypes were characterized by descriptive variables; and no further statistical analysis was requested. Collected information included: patient demographics; type and location of transient osteoporosis, systemic osteoporosis or bone marrow edema; imaging, laboratory and pathology results; clinical features, including pain intensity, tenderness and swelling, movement limitation and limp, and subchondral fractures; risk factors, including pregnancy, precedent trauma and precedent infection; treatment, follow-up and complications. Based on our findings, we also include a proposed management protocol for the various subtypes of TPBMES (Figure 7).

\section{Results}

A review of 546 suspected TPBMES patients was carried out. The proposed classification of TPBMES is listed in Table 1, alongside its differential diagnoses.

Table 1. Transient Primary Bone Marrow Edema Syndrome: classification and differential.
Classification:
Transient osteoporosis of hip (TOH)
TOH of pregnancy
Transient osteoporosis of knee (TOK)
Transient osteoporosis of foot and ankle
Transient bone marrow edema with systemic osteoporosis
Transient regional migratory osteoporosis ("migratory")
Differential diagnosis:
Avascular necrosis of bone (AVN)
Bone contusion
Reflex sympathetic dystrophy
Infection
Rheumatoid arthritis 
Most TPBMES subtypes were found to have several features in common, including: joint pain disproportionate to the physical findings; transient bone marrow edema, unaccounted for by a primary pathology and detected by MRI; a self-limiting clinical course, however protracted it may be. Diagnosis of TPBMES required the exclusion of avascular necrosis, significant bone trauma, infection, reflex sympathetic dystrophy and rheumatoid arthritis. These disease entities may be defined as a non-transient bone marrow edema that is secondary to trauma, infection or other causes. However, systemic osteoporosis has occurred in otherwise typical TPBMES patients. Therefore, transient primary osteoporosis is not ruled out by evidence of systemic osteoporosis. Moreover, the migratory subtype of this disorder develops in a proportion of these patients.

The demographics in Table 2 highlight six subtypes, proposed to constitute the TPBMES disease entity. There were 342 cases of transient osteoporosis of the hip (TOH), mostly in middle-aged males, most of them confirmed by the $\mathrm{MR}$, as soon as this modality was available for imaging. DXA was performed in a minority of these patients. TOH of pregnancy was disclosed in 105 younger women, most of whom in the third trimester of pregnancy. Ten older women were diagnosed with transient osteoporosis of the knee. Footand-ankle transient bone marrow edema is described in 49 patients, mainly females. Some of these cases displayed migratory features.

Table 2. Demographics and clinical studies in TPBMES.

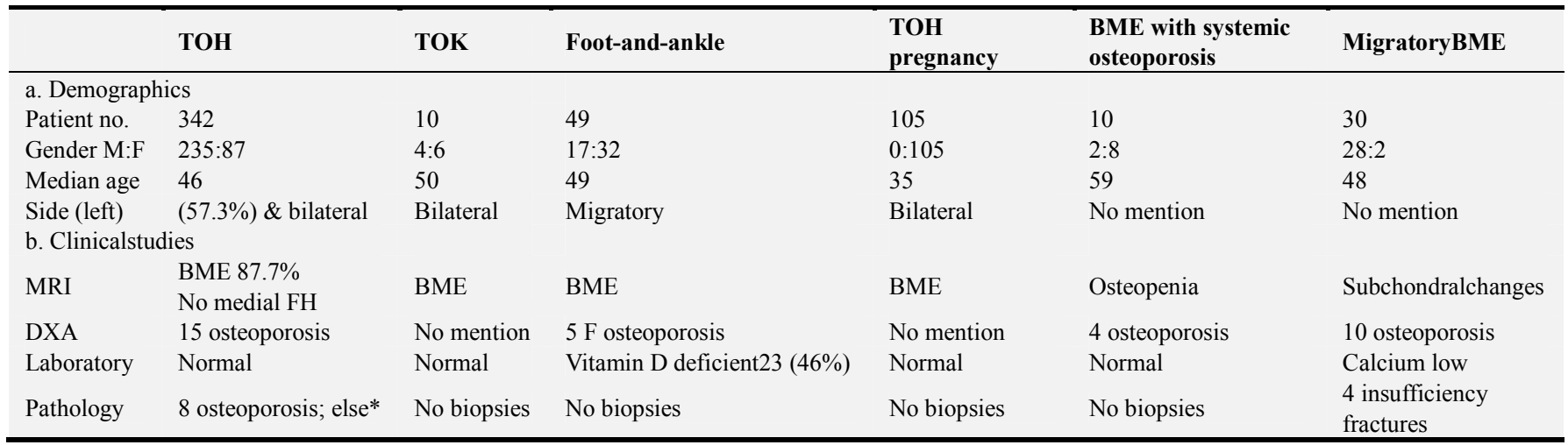

Abbreviations: TOH, transient osteoporosis of hip; TOK, transient osteoporosis of knee; BME, bone marrow edema; FH, femoral head.

* Osteopenia, thin bone seams in marrow spaces, lipid cysts.

In 30 patients, the clinical picture was clearly migratory, of whom a majority were middle-aged males. The development of the migratory subtype was usually delayed. DXAconfirmed systemic osteoporosis was found in 10 patients. A few patients displayed subchondral changes and four, who were submitted to biopsy, displayed insufficiency fractures.
Ten patients, mainly males, had TPBMES with systemic osteoporosis. Intense joint pain, local swelling and subchondral fractures, diagnosed by imaging, were the predominant clinical features, especially in patients with $\mathrm{TOH}$ (Table 3). In addition to pregnancy, suggested risk factors included obesity and vitamin D deficiency.

Table 3. Clinical features and risk factors in TPBMES.

\begin{tabular}{|c|c|c|c|c|c|c|}
\hline & TOH & TOK & Foot and ankle & TOHPregnancy & BME withsystemicosteoporosis & Migratory BME \\
\hline \multicolumn{7}{|l|}{ a. Clinical features: } \\
\hline Pain intensity & +3 & +3 & +2 & +3 & +2 & +2 \\
\hline Limited movement & 3 pts & $1 \mathrm{pt}$ & None & $1 \mathrm{pt}$ & None & $1 \mathrm{pt}$ \\
\hline Limp & $1 \mathrm{pt}$ & $1 \mathrm{pt}$ & None & 2 pts & None & 2 pts \\
\hline Tenderness & $1 \mathrm{pt}$ & 2 pts & None & $1 \mathrm{pt}$ & $1 \mathrm{pt}$ & 3 pts \\
\hline Swelling & $46 \mathrm{pts}$ & None & None & None & Mild & 3 pts \\
\hline b. Risk factors: & & & & & & \\
\hline Pregnancy & $2 \mathrm{pts}$ & None & None & $3^{\text {rd }}$ trimester & None & none \\
\hline Precedent trauma & $6 \mathrm{pts}$ & None & None & None & None & $1 \mathrm{pt}$ \\
\hline Precedent infection & None & None & None & None & None & $1 \mathrm{pt}$ \\
\hline
\end{tabular}

Abbreviations: IF, insufficiency fractures; pt, point

A conservative management was used variably in all the subtypes. Additional modalities of treatment were explored, mainly for the TOH subtype (Table 4). In a few patients, a modern therapeutic modality, namely the intravenous administration of bisphosphonates, may have curbed the symptoms substantially [15-19]. 
Table 4. Therapy and follow-Up of TPBMES.

\begin{tabular}{|c|c|c|c|c|c|c|}
\hline & TOH & TOK & Foot and ankle & TOH of pregnancy & $\begin{array}{l}\text { BME with systemic } \\
\text { osteoporosis }\end{array}$ & Migratory BME \\
\hline \multicolumn{7}{|l|}{ Management, follow-up: } \\
\hline Conservative or none & Variable & Variable & Variable & Variable & Variable & Variable \\
\hline Physicaltherapy, NSAID, steroids & + & None & + & + & None & + \\
\hline Vitamin D, calcium & + & None & + & + & None & + \\
\hline Bisphosphonates & + & None & None & None & None & + \\
\hline Hyperbaric $\mathrm{O}_{2}$ & + & None & None & None & None & None \\
\hline Decompressio & + & None & None & None & None & None \\
\hline Follow-up duration & $1-214 \mathrm{~m}$ & $12-36 \mathrm{~m}$ & $6 \mathrm{~m}$ & $3 \mathrm{~m}$ post- delivery & $5-10 \mathrm{~m}$ & $2 \mathrm{w}-6 \mathrm{~m}$ (up to $3 \mathrm{y}$ ) \\
\hline Complications & Relapse & Relapse & None & Relapse & None & Migratory \\
\hline Progress to migratory & $30(19.4 \%)$ & None & None & None & None & + \\
\hline
\end{tabular}

In a majority of TPBMES cases, the disease was selflimited, lasting from 2 weeks to 10 months. By contrast, in 3 of 6 articles reviewed on compound TPBMES with systemic osteoporosis, the follow-up had lasted intermittently as long as 11 years. A proportion of these patients also developed migratory features (Tables 5,6 ). In these patients the pain was prolonged, albeit with variable asymptomatic pain-free intervals.

Table 5. TPBMES and systemic osteoporosis: demographics and clinical features.

\begin{tabular}{|c|c|c|c|c|c|c|c|c|}
\hline Article no. & $\mathbf{N}$ & GenderM:F & Medianage & Type & DXA & MRI & Subchondralfractures & Bilateral \\
\hline 1 & 141 & $107: 34$ & 46 & $\mathrm{TOH}$ & $15 \mathrm{SOP}$ & BME $87.7 \%$ & $76(48.7 \%)$ & $4 \mathrm{pts}$ \\
\hline 9 & 31 & $11: 20$ & 49 & Migratory knee & $2 \mathrm{SOP}$ & BME & Insufficiency fractures & Yes \\
\hline 12 & 18 & $6: 12$ & 54 & $\mathrm{~F} \& \mathrm{~A}$ & $5 \mathrm{SOP}$ & 18 pts -39 bones & None & Yes \\
\hline 16 & 10 & $2: 8$ & 59 & BME, SOP & 4 SOP & Osteopenia & None & None \\
\hline 19 & 5 & $4: 1$ & $33-62$ & MigratoryBME & $5 \mathrm{SOP}$ & BME subchondral changes & $1 \mathrm{pt}$. & Yes \\
\hline 20 & 3 & $3: 0$ & $43-54$ & MigratoryBME & SOP & No MRI in use & None & Yes \\
\hline
\end{tabular}

Abbreviations: SOP, systemic osteoporosis; F\&A, foot andankle; BME, bone marrow edema.

Table 6. TPBMES and systemic osteoporosis: pathology, outcome and follow-up.

\begin{tabular}{|c|c|c|c|c|c|c|}
\hline Article no. & Pathology & Treatment & Outcome & Follow-up & Progress to migratory & Complications \\
\hline 1 & No biopsy & Not mentioned & SL & $1-10$ years & $30(19.4 \%)$ & None \\
\hline 9 & 4 pts IF & Immobilization & SL & $2 w-6 m$ & None & None \\
\hline 12 & No biopsies & Boots, bisphosphonates & Migratory & $2 y(2-11$ years $)$ & 8 pts & Relapse \\
\hline 16 & No biopsies & Immobilization & SL & $5-10 \mathrm{~m}$ & None & None \\
\hline 19 & 4 biopsies - irrelevant & Ilopost & SL & $9 \mathrm{~m}$ & None & Relapse/migratory \\
\hline 20 & $1 \mathrm{pt}$, focal OP & Physicaltherapy, alendronate & SL & Up to 3 years & Yes & Relapse/migratory \\
\hline
\end{tabular}

Abbreviations: SL, self-limiting disorder; IF, insufficiency fracture; OP, osteoporosis.

In sparse studies, avascular necrosis has been implied to belong to the spectrum of TPBMES. Subchondral fractures were detected in cases of $\mathrm{TOH}$, with or without pregnancy, but they were not considered as indicative of AVN.

\section{Discussion}

The review we carried out seems to support the notion that TPBMES is most probably a single disease, whether it affects the hips $(\mathrm{TOH})$ in middle aged males [1], or in younger females in their last trimester of pregnancy [20, 21]. It may remain the same condition, with mild variations, if it involves the knee (TOK) [9] or the foot-and-ankle [2, 6, 15, 22]. Although it presents with similar features, TPBMES accompanied by systemic osteoporosis, mainly in older males, may acquire a more protracted clinical course $[1,6$, 12]. As these features have no predilection for older women, they must represent a basis divergent from that of primary osteoporosis, especially since a "migratory" pattern of
TPBMES often occurs [23]. Nevertheless, as the clinical features, as well as the self-limiting nature of all the subsets, are analogous, and so are the diagnosis and the resolution of the lesions as confirmed by the MRI, they most probably belong to the same disease entity. Only isolated patients underwent a trephine biopsy. This procedure is not therapeutic and its diagnostic yield is most clearly inferior to that of the MRI.

It is remarkable that patients with protracted disease had either systemic osteoporosis or a migratory subset or both, in addition to features of TPBMES. Notably, most of these patients had $\mathrm{TOH}$. A cause for the longer course in the composite variants has not been assessed so far. Nevertheless, no valid argument is available to establish these subsets as distinct disorders. Since the disorder is selflimiting and, as such, is considered to have an excellent outcome, a lengthy duration of the symptoms might reflect on the patients' condition, mainly that of the inferior part of their skeleton $[1,6,11]$. 
A recurrent problem for the reviews authors was to cope with the complex nomenclature and with the differential diagnosis. Some authors have considered, perhaps inaccurately, avascular necrosis as a part of the TPBMES. Probably because of its rarity, different subsets of the transient bone marrow edema is poorly recognized by physicians and thus needs to be further investigated.

Of particular interest are the differences among transient osteoporosis, systemic osteoporosis and the migratory pattern. That is because of the necessity to try and curb the duration of the symptoms in these protracted forms of the disease. Among the unusual therapy modalities suggested by some authors one of the bisphosphonates, zoledronic acid, was administered in a single $5 \mathrm{mg}$ I. V. infusion, and was reported to cause a remission in a proportion of cases [18,
19]. This medication given in a single dose is also advantageous in terms of the expense. The limitations of this study are listed in Table 8.

\section{Conclusions}

Our analysis suggests that the variously labeled types of transient marrow edema and transient osteoporosis are subtypes of a single disease entity, the TPBMES. While remains self-limiting, TPBMES accompanied by systemic osteoporosis, with or without a migratory pattern, stands out by the prolonged clinical course. Avascular necrosis is excluded from being a TPBMES subtype, as it is neither primary nor transient. A suggested treatment is based on a single $5 \mathrm{mg}$ I. V. dose of zoledronic acid [18, 19].

Table 7. A proposal for the management and follow-up of TPBMES.

As soon as TPBMES is suspected, an MRI must be performed

Less than 8 weeks are necessary to rule out the differential diagnosis or the migratory TPBMES.

During this period, plain X-rays are performed, and calcium, vitamin D and PTH levels are assessed.

At this point, it is suggested to administer calcium, vitamin D and consider 100 IU S. C. $x 2$ calcitonin; physical therapy and NSAID dispensed as necessary

Subsequently, DXA is performed. If migratory TPBMES and/or systemic osteoporosis are diagnosed, the next stage is:

A single I. V. $5 \mathrm{mg}$ dose of zoledronic acid is proposed, administered by infusion, unless contraindicated.

Three months later, after clinical evaluation of the patient, a repeat MRI is done

Depending on the MRI, 3 additional months of follow-up may be necessary.

Table 8. Synthesis limitations.

Studies antedating the introduction of MRI are rare, but do occur

TPBMES is under-diagnosed, because many physicians are unfamiliar with it and it often presents with non-specific symptoms

The classification of the disease entity may seem at times irreproducible

There may be some inconsistencies: TPBMES may be confused with early AVN; the migratory pattern may follow a protracted clinical course; DXA has

been performed erratically

Systemic osteoporosis in this context may be more frequent than is shown in the present study

In spite of the eligibility criteria, a minority of patients were treated with bisphosphonates or calcitonin

\section{Conflict of Interest and Source of Funding}

The authors declare that they have no conflict of interest. No funding was obtained for the completion of this study.

\section{Acknowledgements}

We thank Kibbutz Sde-Boker and Professor Gerard Bueno for their pertinent suggestions.

\section{References}

[1] Klontzas ME, Vassalou EE, Zibis AH, Bintoudi A, Karantanas A. MR imaging of transient osteoporosis of the hip: An update on 155 hip joints. Eur J Radiol 2015;84:431-436.

[2] Wilson AJ, Murphy WA, Hardy DC, Totty WG. Transient osteoporosis: transient bone marrow edema? Radiology1988;167;757-760.

[3] Geith T, Niethammer T, Milz S, Dietrich O, Reiser M, BaurMelnyk A. Transient bone marrow edema syndrome versus osteonecrosis: perfusion patterns at dynamic contrastenhanced MR imaging with high temporal resolution can allow differentiation. Radiology2017;283:478-485.

[4] Lakhanpal S, Ginsburg WW, Luthra HS, Hunder GG. Transient regional osteoporosis. A study of 56 cases and review of the literature. Ann Intern Med 1987;106:444-449.

[5] Malizos KN, Zibis AH, Dailiana Z, Hantes M, Karahalios T, Karantanas AH. MR imaging findings in transient osteoporosis of the hip. Eur J Radiol 2004;50:238-244.

[6] Singh D, Ferrero A, Rose B, Goldberg A, Cullen N. Bone marrow edema syndrome of the foot and ankle. Foot \& Ankle Specialist 2016;9:218-226.

[7] Curtiss PH Jr, Kincaid WE. Transitory demineralization of the hip in pregnancy. A report of three cases. J Bone Joint Surg Am1959;41:1327-33.

[8] Lequesne M. Transient osteoporosis of the hip: a nontraumatic variety of Sudeck's atrophy. Ann Rheum Dis 1968;27:463-71.

[9] Vardi G, and Turner PJ. Transient osteoporosis of the knee. The Knee 2004;11:21.

[10] Balakrishnan A, Schemitsch EH, Pearce D, McKee MD. Distinguishing transient osteoporosis of the hip from avascular necrosis. Can J Surg 2003;46:187-92. 
[11] Trevisan C, Ortolani S, Monteleone M, Marinoni EC. Regional migratory osteoporosis: a pathogenetic hypothesis based on three cases and the review of the literature. Clin Rheumatol 2002;21:418-425.

[12] Karantanas AH, Nikolakopoulos I, Korompilias AV, Apostolaki E, Skoulikaris N, Eracleous E. Regional migratory osteoporosis of the knee: MRI findings in 22 patients and review of the literature. Eur JRadiol 2008;67:34-41.

[13] Smith A, Lopez-Sola M, McMahon K, et al. Multivariate pattern analysis utilizing structural and functional MRI - in individuals with musculoskeletal pain and healthy controls: a systematic review. Sem Arthritis Rheum 2017; 47:418-431.

[14] YamagushiR, Yamamoto T, Motomura G, et al. Radiological morphology variances of transient osteoporosis of the hip. $\mathrm{J}$ Orthop Sci 2017; 22:687-692.

[15] Guler O, Ozyurek S, Cakmak S, Isyar M, Mutlu S, Mahirogullari M. Evaluation of results of conservative therapy in patients with transient osteoporosis of the hip. Acta Orthop Belg 2015;81:420-426.

[16] Horas K, Fraissler L, Maier G, et al. High prevalence of Vitamin D deficiency in patients with bone marrow edema syndrome of the foot and ankle. Foot \& Ankle Int 2017;38:760-766.

[17] Arayssi TK, Tawbi HA, Usta IM, et al. Calcitonin in the treatment of transient osteoporosis of the hip. Sem Arthritis Rheumatism 2003;32:388-397.

[18] Emad Y, Ragab Y, El-Shaarawy N, Rasker JJ. Transient osteoporosis of the hip, complete resolution after treatment with aledronate as observed by MRI description of eight cases and review of the literature. ClinRheumatol 2012;31:16411647.

[19] Flores-Robles BJ, Sanz-SanzJ, Sanabria-Sanchinel AA. Zoledronic acid treatment in primary bone marrow edema syndrome. J Pain Palliat Care Pharmacother2017; 31:52-56.

[20] Goldman GA, Friedman S, Hod M, Ovadia J. Idiopathic transient osteoporosis of the hip in pregnancy. Int J Gynecol Obstet 1994;46:317-320.

[21] Hadji P, Boekhoff J, Hahn M, Hellmeyer L, Hars O, Kyvernitakis I. Pregnancy-associated transient osteoporosis of the hip: results of a case-control study. Arch Osteoporos2017; 12:11. doi:10.1007/s11657-017-0310-y.

[22] Sprinchorn AE, O'Sullivan R, and Beischer AD. Transient bone marrow edema of the foot and ankle and its association with reduced systemic bone mineral density. Foot \& Ankle Int2011;32:508-512.

[23] Toms AP, Marshall TJ, Becker E, Donell ST, Lobo-Mueller EM, Barker T. Regional migratory osteoporosis: a review illustrated by five cases. Clin Radiol 2005;60:425-438. 